\title{
Modelling and Dynamic Response Characteristics Study of a PAM Bionic Kangaroo Leg Suspension
}

\author{
Yong Song, Jiahao Shi, Zhanlong Li, Jinyi Lian, Qinglu Shi and Bijuan Yan \\ School of Mechanical Engineering, Taiyuan University of Science and Technology, Taiyuan 030024, China. \\ No.66 Waliu Road, Wanbailin District, Taiyuan City 030024, Shanxi Province, China.
}

(Received 28 November 2019; accepted 1 April 2020)

\begin{abstract}
A PAM (pneumatic artificial muscle) bionic kangaroo leg suspension is proposed on the basis of a kangaroo leg structure evolved from long-term hopping; the modelling and characteristics research are conducted to pursue a high-performance vehicle suspension system. Based on the PAM and kangaroo leg bone proportions, the bionic suspension structure is constructed by analysing and refining the kangaroo leg structure and functions. The dynamic equations are derived by the Lagrange's Equations considering the rods system features and an Adams simulation model is built up to study the damping performance and parameter characteristics of the suspension. Moreover, a co-simulation of Adams and Matlab is performed under fuzzy control and PID control. The dynamic response characteristics of the suspension is simulated and analysed under the passive and active modes in the time and frequency domains. The result indicates that the vibration and shock of the vehicle body can be reduced effectively by the proposed suspension in passive, fuzzy control and PID control modes; compared with the passive mode, the damping performance of the suspension is better under the active control. The fuzzy control and the PID control are effective to reduce the suspension transmissibility, especially in the medium frequency ranges, and the two control effects are better than that of the passive mode in most frequency bands. The study result of this paper can provide a reference for the research and development of high-performance bionic suspension.
\end{abstract}

\section{INTRODUCTION}

Vehicles as a manned and goods-carrying transportation tool have been greatly improving transportation and work efficiencies, significantly promoting economy development and society progress, and becoming an indispensable part in our daily life and production activities. With the development of science and technology and the progress of human civilization, vehicles will play more important roles. At the same time, the vehicle development and design requirements become more diversified, and the needs of vehicle performances and qualities (such as higher speed, more safety chassis, a more comfortable ride, and more intelligent control) also become more and more rigorous. Especially, the demands of suspension performances are more prominent including handling stability in high speed, passing ability, ride comfort on- and off-road, and so on. Therefore, it has theoretical and engineering values to study and design a new type of suspension, particularly with the characteristics such as intelligent control, quick response, high efficiency, low cost, among others.

Seen from the suspension performances, suspensions can be classified into passive, semi-active, and active suspensions by control mode. ${ }^{1}$ The passive suspension is widely used and its stiffness and damping parameters can be passively adjusted. The adjustment range of this suspension is limited and it cannot meet the operation requirements. However, it has many advantages such as simple structure, low cost, reliable performance, and so on. ${ }^{2}$ The active suspension can satisfy the vehicle demands better through actively adjusting the suspension parameters, but the disadvantages of this suspension are the high technical requirement, the complex structure, and the high cost. ${ }^{3}$ The semi-active suspension can actively adjust the suspension parameters with the less energy input, combining the advantages of the passive and active suspensions. ${ }^{4}$ Plainly, the pursuit of a high quality vehicle puts up a challenge to the suspension design.

Biomimetics is a cross discipline, which combines life science with the disciplines of machinery, materials and information, and so on, providing new ideas, principles and theories for technological innovation and effectively solving many hard engineering problems. ${ }^{5}$ For example, a PAM (pneumatic artificial muscle, a kind of device used to convert the pneumatic pressure into the mechanical force) invented based on muscle characteristics, can be applied to accurate grasping with soft hands. ${ }^{6}$ So, it is reasonable that a higher performance suspension can be designed and developed to satisfy the people's pursuits by applying biomimetic principles and methods.

As is known to all, kangaroos are widely distributed in Australia and can travel in various grounds by jumping. ${ }^{7}$ In kangaroo jumping, it keeps a high speed movement with small energy consumptions for a low requirement of ground, and the jumping is quite steady and almost vibration-less, thanks to its specialized leg structure under the natural long-term hopping. ${ }^{8}$ Therefore, many valuable and interesting studies about kangaroo jumping have been carried out. ${ }^{9-12}$ The Germany Festo company manufactured a kind of bionic kangaroo robot, named Bionic Kangaroo. ${ }^{13}$ The jumping motion control strategies and the experimental researches of the bionic kangaroo were conducted by Knut et al. ${ }^{14}$

In a word, many achievements have been obtained in the research of suspensions and kangaroo jumping. The studies focused on the improvement of the suspension structure and performance, or the jumping and walking functions for kangaroo robot. ${ }^{15-17}$ However, in this paper, these performances such as the cushioning and damping performance of kangaroo leg during landing and take-off, the good road adaptability 


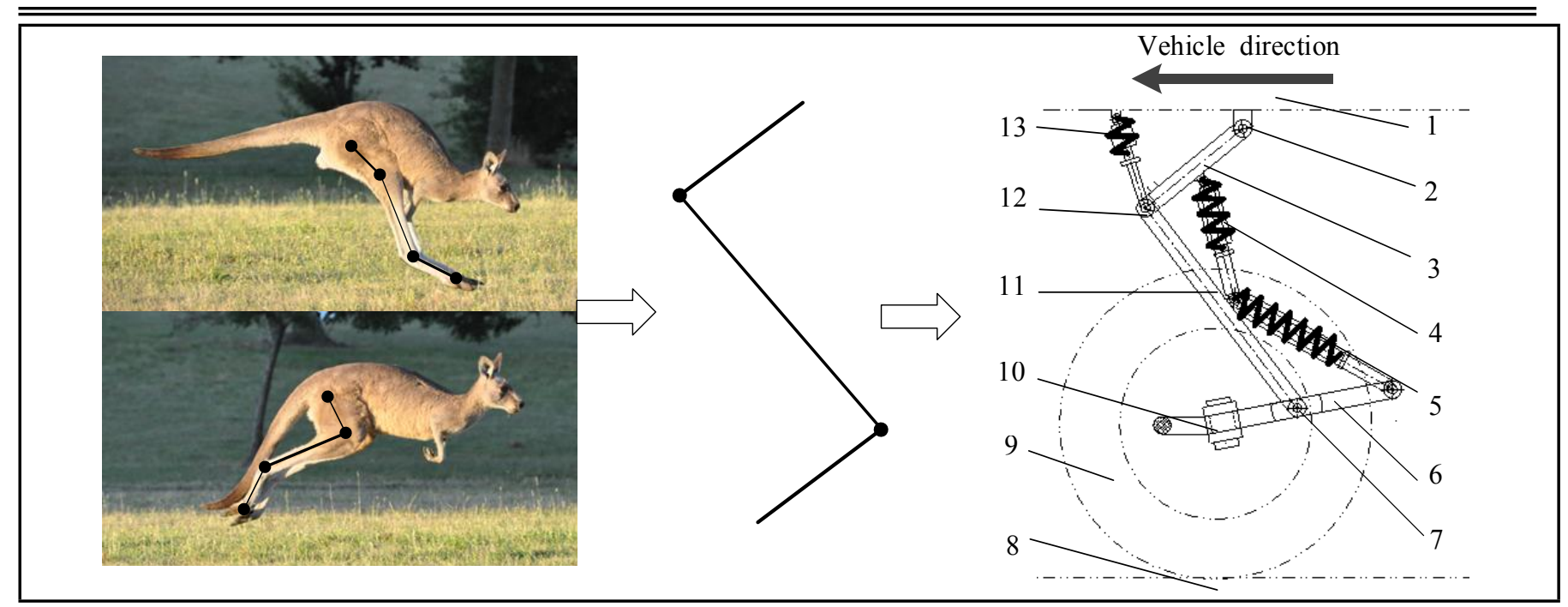

Figure 1. Design ideas and arrangement of the bionic suspension mechanism.

and the high-speed stability during jumping, and so on, were of concern and discussed for the high consistency with the performance requirements of vehicle suspension. Therefore, based on bionic design thinking, the good performances of kangaroo and its jumping structure were considered with the design of suspension structure and performance. By analysing the structure and function of kangaroo leg and applying them to improve the suspension system performances, a new suspension named "PAM bionic kangaroo leg suspension" was designed and studied.

\section{PAM BIONIC KANGAROO SUSPENSION STRUCTURE}

The PAM bionic kangaroo leg suspension was designed by analysing and refining the structure and function of kangaroo legs. ${ }^{18}$ In the suspension, bones were considered as the rods with the same size ratio; the damping function of muscles was treated as the damping effect of dampers and the contractility of muscles was replaced by the contraction force of PAMs; the elastic energy storage function of tendons was substituted by the energy storage function of springs. The bionic suspension structure was constructed by the bionic bones (femur, tibiafibula, and metatarsals), joints (hip, knee, and ankle), muscles (thigh, calf, and gastrocnemius), and tendons (thigh, calf, and foot).

The bones structure of the kangaroo leg was equivalent to a three-rod equal bone proportion structure (femur, tibia-fibula, and metatarsals rods); the kangaroo leg joints (hip, knee, and ankle) were simplified into the plane articulated structures; the functions of the kangaroo leg muscles (thigh, calf, and gastrocnemius) were equivalent to the damping effect and the instantaneous output contraction force, which were imitated by the muscle dampers and the PAMs (thigh, calf, and gastrocnemius), respectively; the elastic energy storage function of the tendons (thigh, calf, and foot) was equivalent to the energy storage function of the springs (thigh, calf, and foot). ${ }^{19}$ The design ideas and arrangement of the bionic suspension mechanism are shown in Fig. 1.

In Fig. 1, 1 is the vehicle body, 2 is the hip joint, 3 is the femur rod, 4 is the combination of bionic calf tendon spring and muscle damper, and the calf PAM, 5 is the combination of

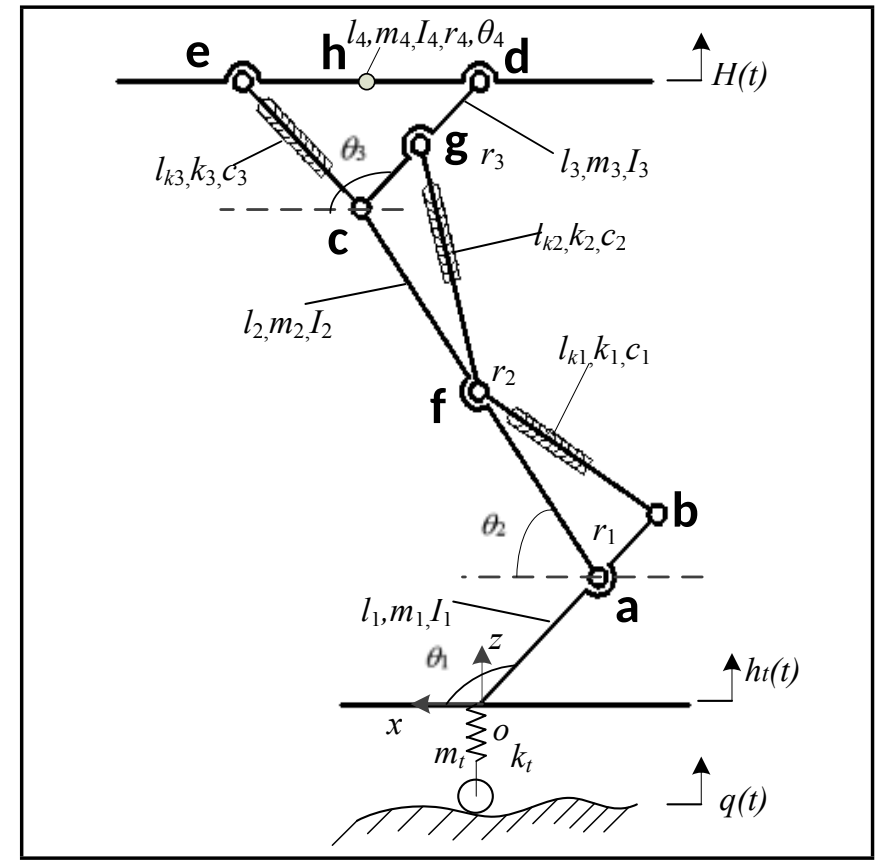

Figure 2. Simplified suspension structure.

bionic foot spring and muscle damper, and the gastrocnemius PAM, 6 is the metatarsals rod, 7 is the ankle joint, 8 is the road surface, 9 is the tire, 10 is the connection between kingpin and wheel, 11 is the tibia-fibula rod, 12 is the knee joint, 13 is the combination of bionic thigh tendon spring and muscle damper, and the thigh PAM. The above combinations were arranged in parallel connection.

To facilitate the dynamic modelling, the structure of the bionic suspension needed to be reasonably simplified and supposed. The conditions were as follows: (1) each bionic bone rod was a rigid body and the centroid was at the geometric centre; (2) space motion of the suspension was treated as plane motion and space rotary motion of the joints was considered as plane rotation. The simplified rod system is shown in Fig. 2.

In Fig. 2, the rod system proportions of the suspension were determined as $1: 2: 1.35\left(l_{\mathrm{dc}}: l_{\mathrm{ca}}: l_{\mathrm{bo}}\right)$ for the lengths of the kangaroo's femur $\left(l_{3}\right)$, tibia-fibula $\left(l_{2}\right)$ and metatarsals $\left(l_{1}\right)$, $l_{4}$ was the simplified vehicle body length; $h, g$ and $f$ were the centres of the rods, $a$ was the point at $2 / 3$ of the rod $b o$ 
$\left(l_{\mathrm{oa}}: l_{\mathrm{ob}}=2: 3\right)$, point $o$ was the equivalent tire enter point; $d$, $c$, and $a$ were the positions of the revolute joints; $\theta_{i}(\mathrm{i}=1,2,3,4)$ was the angle between each rod and the positive $x$-axis; $m_{t}$ was the mass of the tire, $m_{i}(i=1,2,3,4)$ denoted the mass of each rod; $I_{i}(i=1,2,3,4)$ denoted the rotational inertia of each rod; $k_{i}$ was $i$ th spring stiffness; $C_{i}(i=1,2,3)$ was the damping coefficient of $i$ th damper; $q(t)$ represented the pavement excitation, the displacement response of point $o$ to the pavement excitation was denoted as $h_{t}(t), H(t)$ was the body displacement response.

\section{DYNAMIC MODELLING}

The bionic suspension was a complex dynamic system including rod system, bionic joints and other components. In this paper, the kinetic equations of the bionic suspension were established by the Lagrange's equations. ${ }^{20}$ The Lagrange's equations were expressed as:

$$
\frac{d}{d t}\left(\frac{\partial L}{\partial \dot{q}_{i}}\right)-\frac{\partial L}{\partial q_{i}}+\frac{\partial D}{\partial \dot{q}_{i}}=Q_{i}(i=1,2,3, \cdots n)
$$

where $L$ was the Lagrange function, $L=T-V, T$ was the system kinetic energy, $V$ was the system potential energy, $D$ was the system dissipative energy, $q_{i}$ was the generalized $i$ th coordinate, $\dot{q}_{i}$ was the derivative of $q_{i}$, and $Q_{i}$ was the generalized force on $q_{i}$.

The generalized coordinates of the bionic suspension were represented by $\mathbf{q}=\left[\theta_{1}, \theta_{2}, \theta_{3}, \theta_{4}\right]^{T}$ and the generalized forces were represented by $\mathbf{Q}=\left[Q_{1}, Q_{2}, Q_{3}, Q_{4}\right]^{T}$, the absolute coordinate system of $x o z$ and the variable symbols were shown in the dynamic modelling Fig. 2.

The kinetic energy of the bionic suspension system can be derived from the following derivation process: the centroid coordinates of the bionic metatarsals, bionic tibia-fibula and bionic femur rod, and vehicle body were denoted as $\mathbf{r}_{\mathbf{i}}(i=1,2$, $3,4)$, it can be expressed as:

$$
\left\{\begin{array}{l}
\mathbf{r}_{1}=\left[\begin{array}{l}
x_{1} \\
z_{1}
\end{array}\right]=\left[\begin{array}{c}
\frac{1}{2} l_{1} \cos \theta_{1} \\
\frac{1}{2} l_{1} \sin \theta_{1}+h_{t}(t)
\end{array}\right] \\
\mathbf{r}_{2}=\left[\begin{array}{l}
x_{2} \\
z_{2}
\end{array}\right]=\left[\begin{array}{c}
\frac{2}{3} l_{1} \cos \theta_{1}+\frac{l_{2}}{2} \cos \theta_{2} \\
\frac{2}{3} l_{1} \sin \theta_{1}+\frac{t_{2}}{2} \sin \theta_{2}+h_{t}(t)
\end{array}\right] \\
\mathbf{r}_{3}=\left[\begin{array}{l}
x_{3} \\
z_{3}
\end{array}\right]=\left[\begin{array}{c}
\frac{2}{3} l_{1} \cos \theta_{1}+l_{2} \cos \theta_{2}+\frac{l_{3}}{2} \cos \theta_{3} \\
\frac{2}{3} l_{1} \sin \theta_{1}+l_{2} \sin \theta_{2}+\frac{l_{3}}{2} \sin \theta_{3}+h_{t}(t)
\end{array}\right] ; \\
\mathbf{r}_{4}=\left[\begin{array}{l}
x_{4} \\
z_{4}
\end{array}\right]=\left[\begin{array}{c}
\frac{2}{3} l_{1} \cos \theta_{1}+l_{2} \cos \theta_{2}+l_{3} \cos \theta_{3}+\frac{l_{4}}{2} \cos \theta_{4} \\
\frac{2}{3} l_{1} \sin \theta_{1}+l_{2} \sin \theta_{2}+l_{3} \sin \theta_{3}+\frac{l_{4}}{2} \sin \theta_{4}+h_{t}(t)
\end{array}\right]
\end{array}\right.
$$

where $x_{i}(i=1,2,3,4)$ and $z_{i}(i=1,2,3,4)$ represented the centroid position in the $x$ and $z$ directions of the bionic metatarsals rod, bionic tibia-fibula rod and bionic femur rod, and the vehicle body. The velocity of each component can be written as:

$$
v_{i}=\sqrt{\left(\dot{x}_{i}\right)^{2}+\left(\dot{z}_{i}\right)^{2}}
$$

where $\dot{x}_{i}$ and $\dot{z}_{i}$ were the derivation of the centroid of each rod in the $x$ and $z$ directions, $v_{i}(i=1,2,3,4)$ is the velocity of each rod.

The system kinetic energy $T$ consisted of the system translational energy $T_{t}$ and the system rotational energy $T_{r}$, the equa- tion is:

$$
\begin{aligned}
& T=T_{t}+T_{r}=\sum_{i=1}^{4} \frac{1}{2} I_{i} \dot{\theta}_{i}^{2}+\frac{1}{2} m_{t} h_{t}(t)^{2} \\
& \left.+\frac{1}{2} m_{1}\left[\left(\frac{1}{2} l_{1} \dot{\theta_{1}}\right)^{2}+h_{t} \dot{(t}\right)^{2}+l_{1} \dot{\theta_{1}} \cos \theta_{1} h_{t} \dot{(t)}\right] \\
& +\frac{1}{2} m_{2}\left[\begin{array}{l}
\left(\frac{2}{3} l_{1} \dot{\theta_{1}}\right)^{2}+\left(\frac{1}{2} l_{2} \dot{\theta_{2}}\right)^{2}+\frac{2}{3} l_{1} \dot{\theta_{1}} l_{2} \dot{\theta_{2}} \cos \left(\theta_{1}-\theta_{2}\right) \\
+2\left(\frac{2}{3} l_{1} \dot{\theta_{1}} \cos \theta_{1}+\frac{1}{2} l_{2} \dot{\theta_{2}} \cos \theta_{2}\right) h_{t} \dot{(t)}+h_{t}(t)^{2}
\end{array}\right] \\
& +\frac{1}{2} m_{3}\left[\begin{array}{l}
\left(\frac{2}{3} l_{1} \dot{\theta_{1}}\right)^{2}+\left(l_{2} \dot{\theta_{2}}\right)^{2}+\left(\frac{1}{2} l_{3} \dot{\theta_{3}}\right)^{2} \\
+\frac{4}{3} l_{1} \dot{\theta_{1}} l_{2} \dot{\theta_{2}} \cos \left(\theta_{1}-\theta_{2}\right) \\
+\frac{2}{3} l_{1} \dot{\theta_{1}} l_{3} \dot{\theta_{3}} \cos \left(\theta_{1}-\theta_{3}\right)+l_{2} \dot{\theta_{2}} l_{3} \dot{\theta_{3}} \cos \left(\theta_{2}-\theta_{3}\right) \\
+2 h_{t}(t)\left(\frac{2}{3} l_{1} \dot{\theta_{1}} \cos \theta_{1}+l_{2} \dot{\theta_{2}} \cos \theta_{2}+\frac{1}{2} l_{3} \dot{\theta_{3}} \cos \theta_{3}\right) \\
+h_{t} \dot{(}(t)
\end{array}\right] \\
& +\frac{1}{2} m_{4}\left[\begin{array}{l}
h_{t}(t)^{2}+\left(\frac{2}{3} l_{1} \dot{\theta_{1}}\right)^{2}+\left(l_{2} \dot{\theta_{2}}\right)^{2}+\left(l_{3} \dot{\theta_{3}}\right)^{2}+\left(\frac{1}{2} l_{4} \dot{\theta_{4}}\right)^{2} \\
+\frac{4}{3} l_{1} \dot{\theta_{1}} l_{2} \dot{\theta_{2}} \cos \left(\theta_{1}-\theta_{2}\right)+\frac{4}{3} l_{1} \dot{\theta_{1}} l_{3} \dot{\theta_{3}} \cos \left(\theta_{1}-\theta_{3}\right) \\
+\frac{2}{3} l_{1} \dot{\theta_{1}} l_{4} \dot{\theta_{4}} \cos \left(\theta_{1}-\theta_{4}\right)+2 l_{2} \dot{\theta_{2}} l_{3} \dot{\theta_{3}} \cos \left(\theta_{2}-\theta_{3}\right) \\
+l_{2} \dot{\theta_{2}} l_{4} \dot{\theta_{4}} \cos \left(\theta_{2}-\theta_{4}\right)+l_{3} \dot{\theta_{3}} l_{4} \dot{\theta_{4}} \cos \left(\theta_{3}-\theta_{4}\right) \\
+2 h_{t}(t)\left(\frac{2}{3} l_{1} \dot{\theta_{1}} \cos \theta_{1}+l_{2} \dot{\theta_{2}} \cos \theta_{2}+l_{3} \dot{\theta_{3}} \cos \theta_{3}\right. \\
\left.+\frac{1}{2} l_{4} \dot{\theta_{4}} \cos \theta_{4}\right)
\end{array}\right] ;
\end{aligned}
$$

where $\dot{\theta}_{i}(i=1,2,3,4)$ denoted the angular velocity of $\theta_{i}, h_{t}(t)$ was the speed response of point $o$ to the pavement excitation.

The system potential energy $\mathrm{V}$ was composed of the system gravitational potential energy $V_{g}$ and the system elastic potential energy $V_{k}$, can be described as follows:

$$
\begin{aligned}
V & =V_{g}+V_{k}=m_{t} g h_{t}(t)+m_{1} g\left(h_{t}(t)+\frac{1}{2} l_{1} \sin \theta_{1}\right) \\
& +m_{2} g\left(h_{t}(t)+\frac{2}{3} l_{1} \sin \theta_{1}+\frac{1}{2} l_{2} \sin \theta_{2}\right) \\
& +m_{3} g\left(h_{t}(t)+\frac{2}{3} l_{1} \sin \theta_{1}+l_{2} \sin \theta_{2}+\frac{1}{2} l_{3} \sin \theta_{3}\right) \\
& +m_{4} g\left(h_{t}(t)+\frac{2}{3} l_{1} \sin \theta_{1}+l_{2} \sin \theta_{2}+l_{3} \sin \theta_{3}+\frac{1}{2} l_{4} \sin \theta_{4}\right) \\
& +\frac{1}{2} k_{t}\left(h_{t}(t)-\Delta x_{t}\right)^{2}+\frac{1}{2} k_{1}\left(l_{k 1}-l_{k 10}+\Delta x_{1}\right)^{2} \\
& +\frac{1}{2} k_{2}\left(l_{k 2}-l_{k 20}-\Delta x_{2}\right)^{2}+\frac{1}{2} k_{3}\left(l_{k 3}-l_{k 30}-\Delta x_{3}\right)^{2}
\end{aligned}
$$

where $k_{t}$ was the tire equivalent stiffness, $\Delta x_{t}$ was the deformation of tire in the state of static balance, $\Delta x_{i}(i=1,2,3)$ denoted the deformation of the bionic foot, calf, and thigh springs in the state of static balance, $l_{k 1}, l_{k 2}$ and $l_{k 3}$ were the length of bionic foot, calf, and thigh springs, $l_{k 10}, l_{k 20}$ and $l_{k 30}$ were the length in the state of static balance.

The system dissipative energy $D$ was made up of each mus- 
cle damper dissipative energy, and it can be derived as follows:

$$
\begin{aligned}
D & =\frac{1}{2} C_{1} \Delta{\dot{l_{k 1}}}^{2}+\frac{1}{2} C_{2} \Delta \dot{l}_{k 2}{ }^{2}+\frac{1}{2} C_{3} \Delta \dot{l}_{k 3}{ }^{2} \\
& =\frac{C_{1}\left[l_{1} l_{2} \sin \left(\theta_{1}-\theta_{2}\right)\left(\dot{\theta_{1}}-\dot{\theta_{2}}\right)\right]^{2}}{8 l_{1}{ }^{2}+18 l_{2}{ }^{2}-24 l_{1} l_{2} \cos \left(\theta_{1}-\theta_{2}\right)} \\
& +\frac{C_{2}\left[l_{2} l_{3} \sin \left(\theta_{2}-\theta_{3}\right)\left(\dot{\theta_{2}}-\dot{\theta_{3}}\right)\right]^{2}}{8 l_{2}{ }^{2}+8 l_{3}^{2}+16 l_{2} l_{3} \cos \left(\theta_{2}-\theta_{3}\right)} \\
& +\frac{C_{3}\left[l_{3} l_{4} \sin \left(\theta_{3}-\theta_{4}\right)\left(\dot{\theta_{3}}-\dot{\theta_{4}}\right)\right]^{2}}{2 l_{3}{ }^{2}+2 l_{4}{ }^{2}+4 l_{3} l_{4} \cos \left(\theta_{3}-\theta_{4}\right)}
\end{aligned}
$$

where $\Delta \dot{l_{\mathrm{ki}}}(i=1,2,3)$ denoted the derivative of $\Delta l_{\mathrm{ki}}$.

The generalized force equation of each joint was derived by the Lagrange's Equations for the suspension. Substituting the Eqs. (4), (5) and (6) into Eq. (1), the generalized force equations of the generalized coordinates were obtained. Considering the specific derivation process of the total kinetic energy, potential energy, and dissipative energy of the bionic suspension as cumbersome and lengthy, it is not given here, only the generalized force equation $\left(Q_{4}\right)$ of the generalized coordinate $\theta_{4}$ is given in this paper, the generalized force equation is as follows:

$$
\begin{aligned}
Q_{4} & =\frac{d}{d t}\left(\frac{\partial L}{\partial \dot{q}_{4}}\right)-\frac{\partial L}{\partial q_{4}}+\frac{\partial D}{\partial \dot{q}_{4}} \\
& =I_{4} \ddot{\theta_{4}}+\frac{1}{3} m_{4} l_{1} l_{4} \cos \left(\theta_{1}-\theta_{4}\right) \ddot{\theta_{1}} \\
& +\frac{1}{2} m_{4} l_{2} l_{4} \cos \left(\theta_{2}-\theta_{4}\right) \ddot{\theta_{2}}+\frac{1}{2} m_{4} l_{3} l_{4} \cos \left(\theta_{3}-\theta_{4}\right) \ddot{\theta_{3}} \\
& +\frac{1}{2} m_{4} l_{4}{ }^{2} \ddot{\theta_{4}}-\frac{1}{3} m_{4} l_{1} l_{4} \sin \left(\theta_{1}-\theta_{4}\right) \dot{\theta}_{1}{ }^{2} \\
& -\frac{1}{2} m_{4} l_{2} l_{4} \sin \left(\theta_{2}-\theta_{4}\right) \dot{\theta}_{2}^{2}-\frac{1}{2} m_{4} l_{3} l_{4} \sin \left(\theta_{3}-\theta_{4}\right) \dot{\theta}_{3}{ }^{2} \\
& +\frac{1}{2} m_{4} l_{4} \cos \theta_{4} h_{t}(t)+\frac{1}{2} m_{4} g l_{4} \cos \theta_{4} \\
& +\frac{1}{l_{k 3}} k_{3} l_{3} l_{4} \sin \left(\theta_{3}-\theta_{4}\right)\left(l_{k 3}-l_{k 30}-\Delta x_{3}\right) \\
& -\frac{C_{3}\left[\left(l_{3} l_{4}\right)^{2}\left(\theta_{3}-\theta_{4}\right)\left(\dot{\theta}_{3}-\dot{\theta}_{4}\right)\right]}{l_{3}{ }^{2}+l_{4}{ }^{2}+2 l_{3} l_{4} \cos \left(\theta_{3}-\theta_{4}\right)} ;
\end{aligned}
$$

where $\ddot{\theta}_{i}(i=1,2,3,4)$ denoted the angular acceleration velocity of $\theta_{i}, h(t)$ was the second derivative of the $h(t)$.

Through the dynamic modelling of the PAM bionic kangaroo leg suspension, it can lay the foundation for the trajectory planning and intelligent control of the suspension, and provide theoretical basis for the forward and inverse kinematics problems of the bionic kangaroo leg suspension.

\section{SIMULATION MODELLING}

\subsection{PAM Structure and Output Force}

A PAM is a type of bionic pneumatic actuator. The basic structure of the PAM was a support material that restricted deformation on the outside, and an elastic thin-walled bag inside, the two ends of the bag and the support material were fixed with connectors. When the thin-walled bag of the PAM was

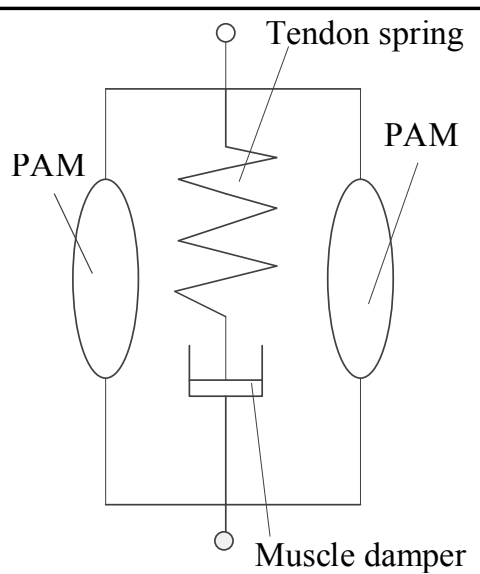

Figure 3. Simplified structure of the combination.

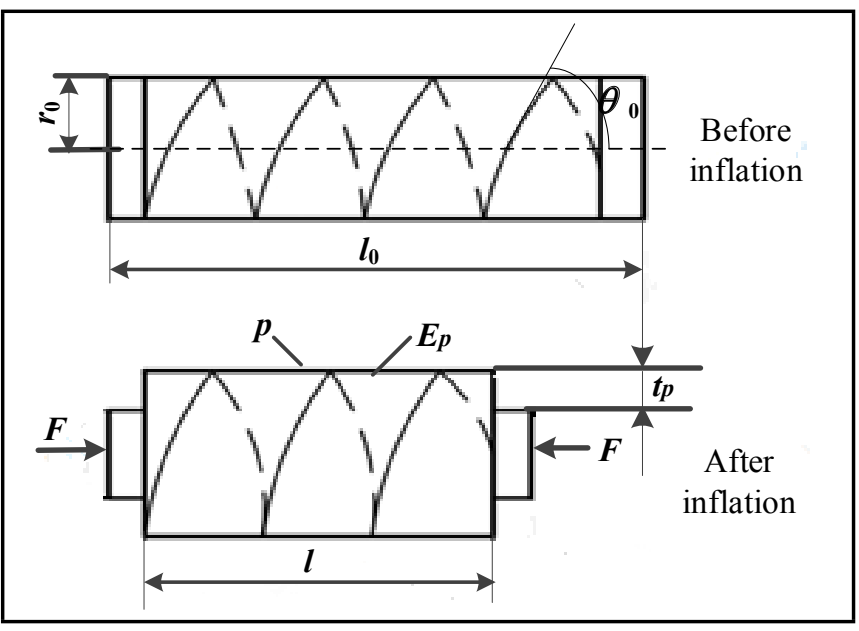

Figure 4. PAM structure.

inflated and pressurized, due to the limitation of the weaving net, it generated axial contraction and axial contraction force. ${ }^{21}$ PAMs have the characteristics of large output force, good dynamic characteristics and lightness, they have similar characteristics to biological muscles in the aspects of performance and structure, which is in line with the bionics idea of this paper. Therefore, the PAMs were selected as the main power output element of the suspension system, its simplified arrangement diagram in the bionic kangaroo leg suspension is shown in Fig. 3.

The working principle of the PAM in the bionic kangaroo leg suspension was: when the contraction force was required between the rods at both ends of the PAM, the PAM generated the contraction force by increasing the air pressure inside the PAM; when the tensile force was needed, the gas in the PAM was released, and the spring force generated by the PAM compression was used as the tensile force. In the active mode of the PAM bionic kangaroo leg suspension, the angles of the bionic hip, knee, and ankle joints were adjusted by controlling the output force of the PAM between the rods, thereby adjusting the posture of the suspension. The magnitude and direction of the output force was determined by adjusting the internal pressure of the PAM and the timing of releasing the gas in the PAM. In this paper, the final output force of the PAM system was used as the control target to achieve self-adjustment of the suspension in the active mode. The PAM structure is shown in Fig. $4 .{ }^{22}$ 


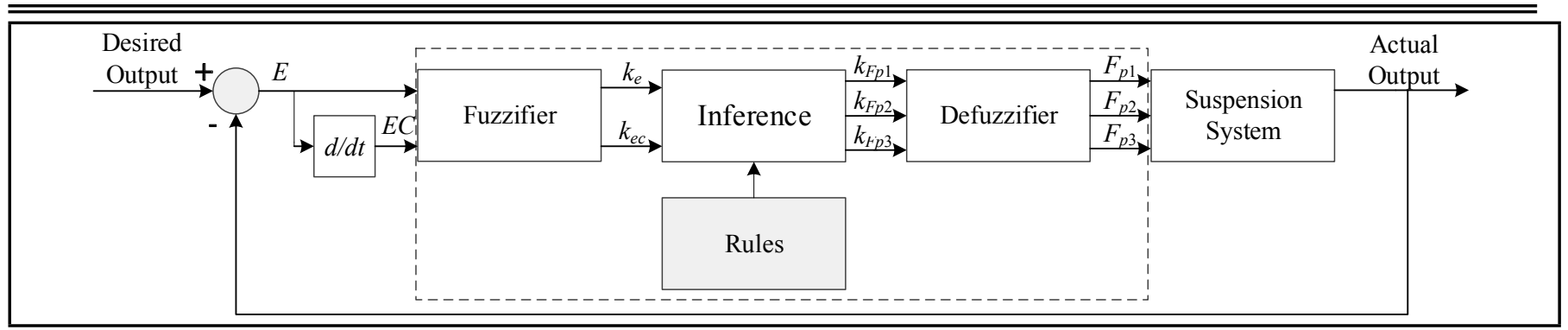

Figure 5. Block diagram of fuzzy controller implementation.

In this suspension design, the PAM was equivalent to a variable stiffness actuator and the functional relationship of its theoretical axial output force $F$ is presented as:

$$
F(\epsilon, p)=\pi r_{0}^{2} p\left[a(1-\epsilon)^{2}-b\right]
$$

where $\epsilon$ was contraction, $\epsilon=\left(l_{0}-l\right) / l_{0}, a=3 / \tan ^{2} \theta_{0}, b=$ $1 / \sin ^{2} \theta_{0}, r_{0}$ and $l_{0}$ represented the initial radius and initial length of the artificial muscle, $\theta_{0}$ represented the initial angle between the artificial mesh and the artificial muscle axis, $l$ was the current length of the artificial muscle; $p$ was the relative pressure inside the artificial muscle.

Moreover, considering the interaction between the internal structures of the PAM during deformation and assuming that the static friction remained unchanged during the motion, the following formula simultaneously expressed the output force ${ }^{23}$ model of artificial muscle under contraction and extension conditions:

$$
\left\{\begin{array}{l}
F=k_{0} \pi r_{0}^{2} p^{\prime}\left[a\left(1-k_{1} \epsilon\right)^{2}-b\right]-f_{t}-F_{r} \operatorname{sign} \dot{\epsilon} \\
f_{t}=\frac{2 \sqrt{3 a}}{2} l_{0} \pi r_{0} t_{p} E_{p}(1-\epsilon)^{2}\left(1-\sqrt{\frac{3}{3 b-a(1-\epsilon)^{2}}}\right)
\end{array} ;\right.
$$

where $k_{0}, k_{1}$ were the correction factors, $F_{r}$ was the friction force during artificial muscle movement, $t_{p}$ represented the wall thickness of the rubber tube, $E_{p}$ was the elastic coefficient of the rubber material. With reference to relevant literatures, the value of the correction factors were determined as $k_{0}=0.7984, k_{1}=1.2942 .{ }^{24}$

Table 1. PAM parameters.

\begin{tabular}{|l|l|}
\hline Parameters & Values \\
\hline$r_{0}$ & $10 \mathrm{~mm}$ \\
$l_{0}$ & $120 \mathrm{~mm}, 220 \mathrm{~mm}, 250 \mathrm{~mm}$ \\
$\theta_{0}$ & $68^{\circ}$ \\
$a$ & 0.6523 \\
$b$ & 1.1632 \\
$F_{r}$ & $54.5 \mathrm{~N}$ \\
$t_{p}$ & $3 \mathrm{~mm}$ \\
$E_{p}$ & $0.00784 \mathrm{GPa}$ \\
\hline
\end{tabular}

In this paper, the DMSP-20 series pneumatic artificial muscle of FESTO Company was used as the main power output element of the bionic kangaroo leg suspension. The specific parameters are listed in Tab. $1 .^{25}$

The detailed PAM parameters used in bionic kangaroo leg suspension were as follows:

All $r_{0}$ values were $10 \mathrm{~mm}$. The lengths of the PAMs at thigh, calf, and gastrocnemius are $120 \mathrm{~mm}, 220 \mathrm{~mm}$ and $250 \mathrm{~mm}$, respectively. The other parameters are the same as in Tab. 1.

\subsection{Fuzzy Control}

The fuzzy control is a modern and flexible control method in the field of control engineering. Considering the performance

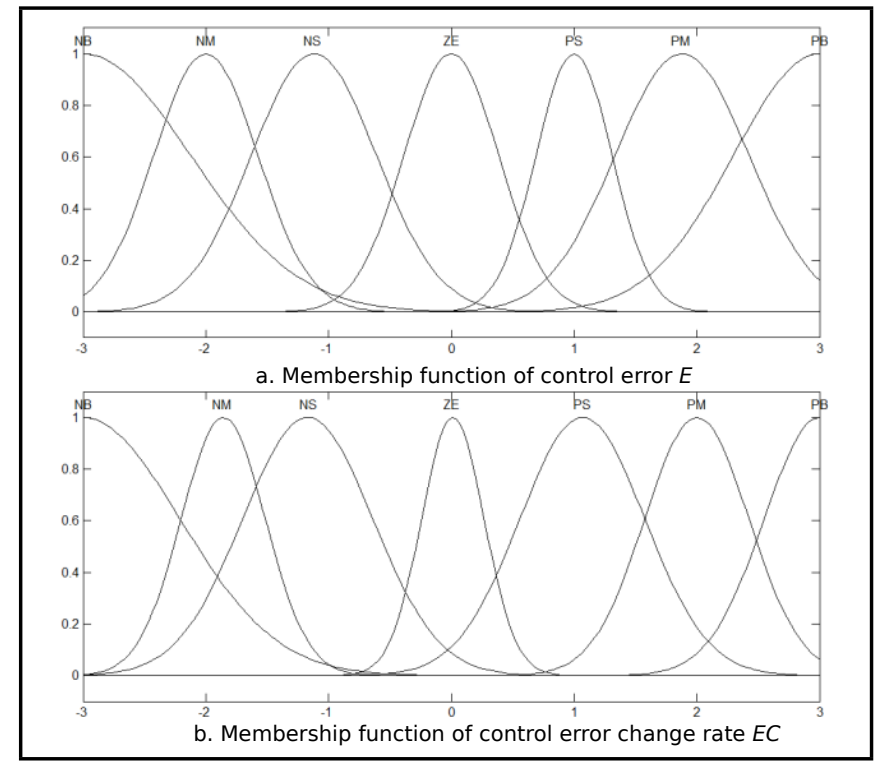

Figure 6. Membership functions of $\mathrm{E}$ and $\mathrm{EC}$.

requirements and control characteristics of the bionic suspension system, the Mamdani-type two-dimensional fuzzy controller was selected, and the block diagram for fuzzy controller implementation is given in Fig. 5. ${ }^{26}$ The inputs of the fuzzy control system were control error $E$ of the vehicle body, vertical vibration acceleration $a_{b}$ and its change rate $E C$, and the control force $U\left(F_{p 1}, F_{p 2}, F_{p 3}\right)$ of the PAM was the output of the fuzzy control system, where $F_{p 1}, F_{p 2}$, and $F_{p 3}$ were the PAM output forces represented by the No. 5, No. 4, and No. 13 components in Fig. 1, respectively. The output force formula of the PAM is given in Eq. (9).

The quantificational universe of the input and output variables is:

$$
E, E C, F_{p 1}, F_{p 2}, F_{p 3}=\{-3,-2,-1,0,1,2,3\} \text {. }
$$

The fuzzy languages to describe $E, E C, F_{p 1}, F_{p 2}$ and $F_{p 3}$ were defined as negative large, negative medium, negative small, zero, positive small, positive medium, and positive large \}, the fuzzy language universes are:

$$
E, E C, F_{p 1}, F_{p 2}, F_{p 3}=\{\mathrm{NB}, \mathrm{NM}, \mathrm{NS}, \mathrm{ZE}, \mathrm{PS}, \mathrm{PM}, \mathrm{PB}\} ;
$$

The control error $E$ was the error between the vehicle body acceleration and the desired acceleration, the desired acceleration was set to $0 \mathrm{~m} \cdot \mathrm{s}^{-2}$, the vehicle body acceleration was obtained by the simulation of the PAM bionic kangaroo leg suspension in the passive mode. In order to improve the control precision, some extreme acceleration values were excluded. 


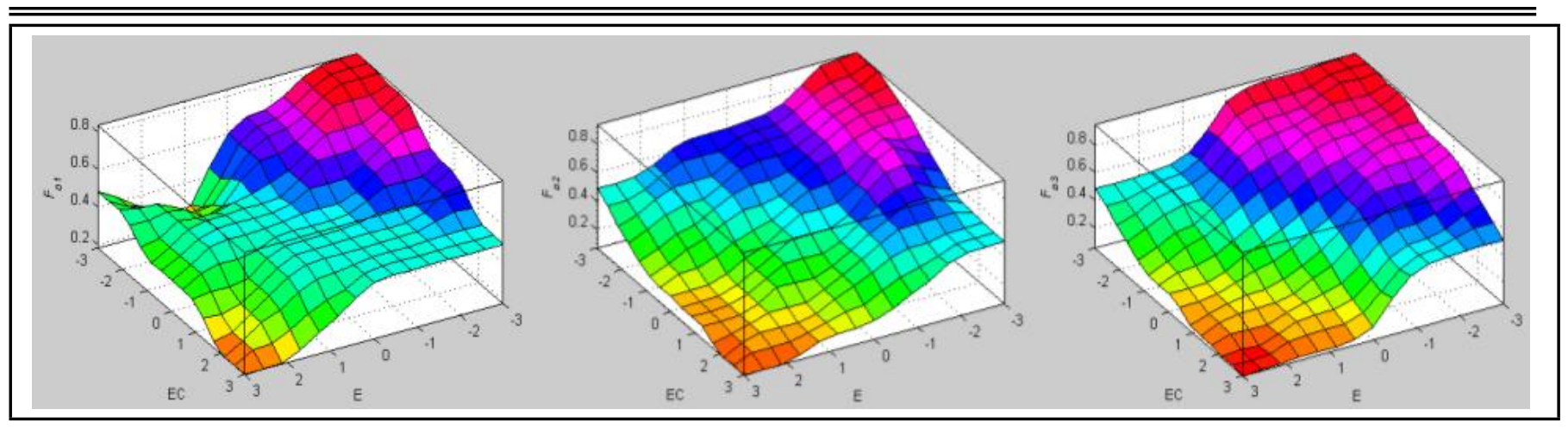

Figure 7. Input and output variables relationship between surface charts.

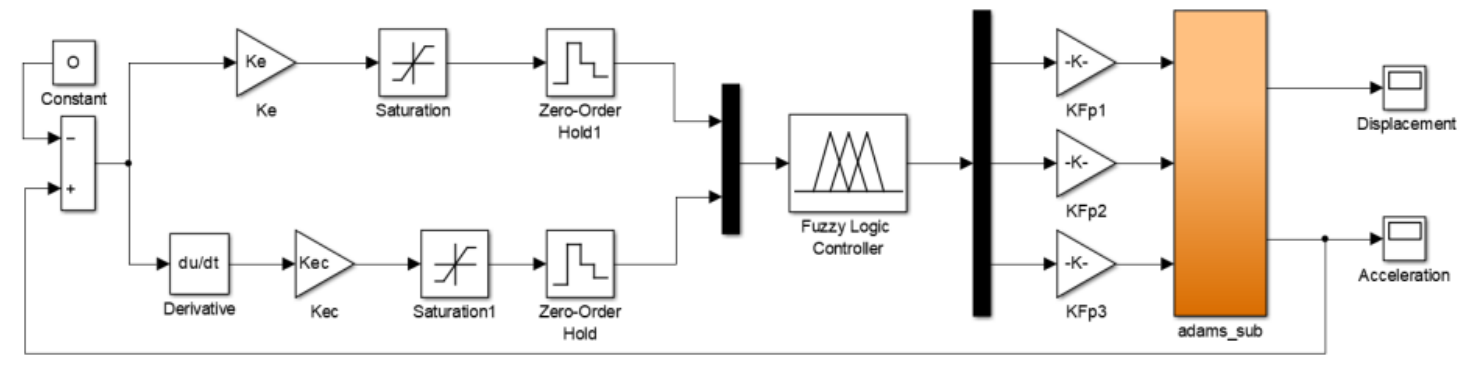

Figure 8. Simulation model of fuzzy control.

The universe of actual physical input $E$ was $[-10,10] \mathrm{m} \cdot \mathrm{s}^{-2}, E C$ is [-6,6]\%, and the actual physical output universes of $F_{p 1}, F_{p 2}$ and $F_{p 3}$ were $[-750,750] \mathrm{N},[-200,200] \mathrm{N}$ and $[-850,850] \mathrm{N}$, respectively. The scaling factors of the fuzzy controller are: ${ }^{27}$

$$
\begin{aligned}
K_{e} & =0.3, \quad K_{\mathrm{ec}}=0.5, \quad K_{F_{p 1}}=250, \\
K_{F_{p 2}} & =66.7, \quad K_{F_{p 3}}=283.3 .
\end{aligned}
$$

In order to facilitate the calculation and maintain a high control resolution, the input variable control error $E$ and its change rate $E C$ of the fuzzy controller were expressed by the Gaussian membership functions, and the output variable $U\left(F_{p 1}\right.$, $\left.F_{p 2}, F_{p 3}\right)$ was represented by the triangle membership functions. Their membership functions are shown in Fig. 6.

To ensure the performance of the fuzzy controller, the following principles were used to design the fuzzy control rules: ${ }^{28}$ (a) when the control error $E$ and its change rate $E C$ were positive large $(P B)$ or positive medium $(P M)$, the selection of the output control variable should be aimed at eliminating the error of the bionic suspension system as soon as possible; (b) when the control error $E$ and its change rate $E C$ were positive small $(P S)$ or zero $(Z E)$, the output control variable should be selected to ensure the stability of the bionic suspension system and prevent the overshoot of the system; (c) when the control error $E$ and its change rate $E C$ were negative, the principle was the same as the positive, and the corresponding symbols changed accordingly. In addition, the difference arrangement positions and the output ranges of the PAMs should be considered for the output control variable $U\left(F_{p 1}, F_{p 2}, F_{p 3}\right)$.

According to the above, a fuzzy control rule table describing the relationship between $E, E C, F_{p 1}, F_{p 2}$ and $F_{p 3}$ was established, as shown in Tab. 2.

Calling the Fuzzy toolbox in Matlab/Simulink, the input and output variable surfaces of the fuzzy controller were obtained, shown in Fig. 7.

\begin{tabular}{|c|c|c|c|c|c|c|c|c|}
\hline & \multicolumn{8}{|c|}{$E C$} \\
\hline \multirow{22}{*}{$E$} & & NB & NM & NS & $\mathrm{ZE}$ & PS & PM & PB \\
\hline & & PM & PM & PM & PS & PS & $\overline{\mathrm{ZE}}$ & $\mathrm{ZE}$ \\
\hline & NB & PB & PB & PM & PS & ZE & $\mathrm{ZE}$ & ZE \\
\hline & & PB & PB & PB & PM & PM & PS & ZE \\
\hline & & PM & $\mathrm{PM}$ & PS & PS & $\overline{\mathrm{ZE}}$ & $\mathrm{ZE}$ & $\mathrm{ZE}$ \\
\hline & NM & PB & PM & PM & PM & PS & $\mathrm{ZE}$ & ZE \\
\hline & & PB & PB & PM & PM & PS & $\mathrm{ZE}$ & ZE \\
\hline & & PS & PS & PS & $\mathrm{ZE}$ & $\mathrm{ZE}$ & $\mathrm{ZE}$ & $\mathrm{ZE}$ \\
\hline & NS & PS & PS & PS & ZE & ZE & $\mathrm{ZE}$ & NS \\
\hline & & PB & PM & PM & PS & PS & $\mathrm{ZE}$ & ZE \\
\hline & & PS & PS & $\overline{\mathrm{ZE}}$ & $\mathrm{ZE}$ & $\mathrm{ZE}$ & $\overline{\mathrm{ZE}}$ & $\mathrm{ZE}$ \\
\hline & $\mathrm{ZE}$ & PS & PS & ZE & ZE & NS & NS & NM \\
\hline & & PB & PM & PS & ZE & NS & $\mathrm{NM}$ & NB \\
\hline & & NM & NS & $\overline{\mathrm{ZE}}$ & $\mathrm{ZE}$ & $\mathrm{ZE}$ & $\overline{\mathrm{ZE}}$ & NS \\
\hline & PS & PS & $\mathrm{ZE}$ & ZE & NS & NS & $\mathrm{NM}$ & NM \\
\hline & & ZE & ZE & $\mathrm{ZE}$ & NS & NM & NM & NB \\
\hline & & NS & $\mathrm{ZE}$ & $\overline{\mathrm{ZE}}$ & $\mathrm{ZE}$ & $\mathrm{NS}$ & $\mathrm{NS}$ & $\mathrm{NM}$ \\
\hline & PM & ZE & ZE & NS & NM & NM & NM & NB \\
\hline & & ZE & ZE & NS & NM & NM & NB & NB \\
\hline & & $\mathrm{ZE}$ & $\mathrm{ZE}$ & $\mathrm{NS}$ & NS & NS & $\mathrm{NM}$ & $\mathrm{NM}$ \\
\hline & PB & ZE & NS & NM & NM & NM & NB & NB \\
\hline & & ZE & NS & NM & NM & NB & NB & NB \\
\hline
\end{tabular}

Table 2. Fuzzy control rules of $F_{p 1}, F_{p 2}$, and $F_{p 3}$.

Mamdani min-max method was adopted to obtain the total control rules, and the centroid defuzzification method was used to get the physical output, its expression is:

$$
U^{*}=\frac{\sum_{i=1}^{n} U_{i} \mu\left(U_{i}\right)}{\sum_{i=1}^{n} \mu\left(U_{i}\right)}
$$

where $U^{*}$ was the physical output of $U, n$ was the quantity of fuzzy relationships, $\mu$ was the membership function of $U, U_{i}$ was the linguistic level of $U$.

Based on the above analysis of the basic principles of fuzzy control, the fuzzy control simulation model of the active suspension system was built in Matlab/Simulink environment, as shown in Fig. 8. 


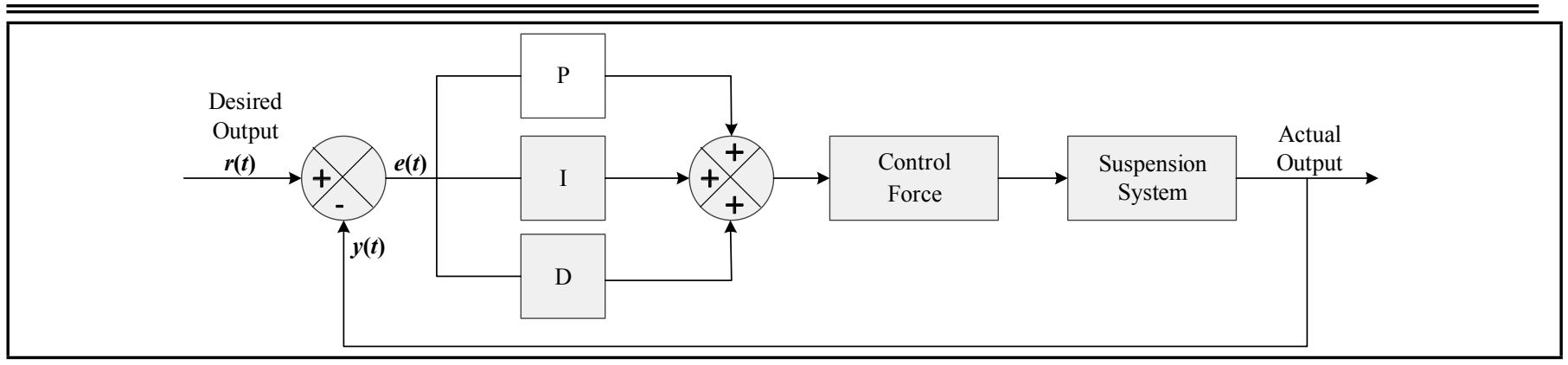

Figure 9. Block diagram of PID controller implementation.

\subsection{PID Control}

PID control is a control strategy that directly controls numbers, where PID is the abbreviation of Proportional, Integral, and Derivative. ${ }^{29}$ It controls the system's tracking error value (the error between the desired output value and the actual output value) by using the operations of $\mathrm{P}$ (proportional), $\mathrm{I}$ (integral) and $\mathrm{D}$ (derivative), so as to make the actual output value as close as possible to the desired value, the block diagram for PID controller implementation is given in Fig. 9.

The tracking error is computed as:

$$
e(t)=r(t)-y(t) ;
$$

where $e(t)$ was tracking error, $r(t)$ was desired output, $y(t)$ was actual output. The mathematical expression of the PID control principle is described as:

$$
u(t)=K_{p} e_{(t)}+K_{i} \int e_{(t)} \mathrm{dt}+K_{d} \frac{d e_{(t)}}{\mathrm{dt}} .
$$

For this paper, the error between the vehicle body acceleration and the desired acceleration of the bionic kangaroo leg suspension was used as the input of the PID control system, and the desired acceleration value was set to 0 , the output forces $F_{p 11}, F_{p 22}$ and $F_{p 33}$ of the PAM system were used as the output of the PID controller, the output force acted on the bionic kangaroo suspension to adjust the suspension.

PID parameter tuning methods mainly include the theoretical calculation method, Niegler-Nichols tuning method, critical proportioning method, etc. The parameters obtained by these methods often need to be further adjusted, which is inconvenient. In this paper, MATLAB/Simulink was used for simulation design, referring to the performance index requirements of bionic kangaroo leg suspension, and adjusted the PID parameters by observing the corresponding response curve. The specific ideas are as follows: ${ }^{30}$

(1) Adjust the Proportional (P) part first. Under the condition that $K_{i}$ and $K_{d}$ are both zeros and remain unchanged, gradually adjust the $K_{p}$ from a small value to a large value until encountering a fast suspension response and slight overshoot, then the $K_{p}$ can be obtained in the Simulink module.

(2) The second step is to adjust the Integral (I) part. First give $K_{i}$ a smaller value, slightly reduce the $K_{p}$ obtained in the first step, and then gradually adjust the $K_{i}$ from a small value to a large value until getting the response curve that can obviously eliminate the offset.

(3) The third step is to adjust the Derivative (D) part. The $K_{i}$ and $K_{p}$ obtained in the second step are reduced slightly, and then the $K_{d}$ is adjusted from a small value to a large value. At the same time, make small adjustments to $K_{i}$ and $K_{p}$ until the response curve can improve the transient response.
Table 3. PID tuning parameters.

\begin{tabular}{|l|l|l|l|l|l|l|l|l|l|}
\hline Parameters & $K_{p 1}$ & $K_{i 1}$ & $K_{d 1}$ & $K_{p 2}$ & $K_{i 2}$ & $K_{d 2}$ & $K_{p 3}$ & $K_{i 3}$ & $K_{d 3}$ \\
\hline Values & 120 & 3000 & 6 & 10 & 1600 & 15 & 150 & 5800 & 4 \\
\hline
\end{tabular}

Table 4. The bionic suspension simulation parameters.

\begin{tabular}{|l|l|}
\hline Parameters & Values \\
\hline $\begin{array}{l}\text { The mass of } m_{1}, m_{2}, m_{3} \text { and sprung mass } \\
m_{4} /(\mathrm{kg})\end{array}$ & $6,8,4,450$ \\
\hline The length of $l_{1}, l_{2}, l_{3}, l_{4} /(\mathrm{m})$ & $0.28,0.42,0.21,0.26$ \\
\hline $\begin{array}{l}\text { The spring stiffnesses of bionic foot, calf and } \\
\text { thigh } k_{1}, k_{2}, k_{3} /\left(\times 10^{4} \mathrm{~N} \cdot \mathrm{m}^{-1}\right)\end{array}$ & $3.18,1.26,3.84$ \\
\hline $\begin{array}{l}\text { The damping coefficients of bionic foot, calf and } \\
\text { thigh } c_{1}, c_{2}, c_{3} /\left(\mathrm{N} \cdot \mathrm{s} \cdot \mathrm{m}^{-1}\right)\end{array}$ & $2276,1451,2498$ \\
\hline $\begin{array}{l}\text { The initial angles of bionic toe, ankle, } \mathrm{knee}, \text { and } \\
\text { hip joints } \theta_{1}, \theta_{2}, \theta_{3}, \theta_{4} /\left(^{\circ}\right)\end{array}$ & $159.4,56.3,156.5,0$ \\
\hline The rotational inertia of $I_{1}, I_{2}, I_{3}, I_{4} /\left(\mathrm{kg} \cdot \mathrm{m}^{2}\right)$ & $0.12,0.05,0.47,0.15$ \\
\hline $\begin{array}{l}\text { The height of vehicle body centroid in static } \\
\text { equilibrium position } \mathrm{H}_{0} /(\mathrm{m})\end{array}$ & 0.50 \\
\hline
\end{tabular}

Table 5. Ranges of joint angle parameters.

\begin{tabular}{|l|l|l|l|l|l||}
\hline Bionic joints & \multicolumn{2}{|l|}{ Compression limit } & Static state & \multicolumn{2}{|c|}{ Tension limit } \\
\hline Bionic hip joint & $\theta_{1}$ & $168.5^{\circ}$ & $159.4^{\circ}$ & $\theta_{1} *$ & $137^{\circ}$ \\
Bionic knee joint & $\theta_{2}$ & $52.8^{\circ}$ & $56.3^{\circ}$ & $\theta_{2} *$ & $64.2^{\circ}$ \\
Bionic ankle joint & $\theta_{3}$ & $162.7^{\circ}$ & $156.5^{\circ}$ & $\theta_{3} *$ & $138^{\circ}$ \\
\hline
\end{tabular}

Because the bionic kangaroo leg suspension has three PAM output force devices, the influence between the three PAM output forces should also be considered when tuning the parameters. The weights of the three output forces affecting the bionic kangaroo suspension are arranged in descending order: Fp3, Fp1, Fp2. The final tuning parameters are shown in Tab. 3:

The PID control simulation model of the active suspension system was built in MATLAB/Simulink environment, as shown in Fig. 10.

\subsection{Simulation Parameters and Model}

The medium and heavy off-road vehicles were taken as the reference objects for the design of the suspension structure. The static balance height of the suspension was assumed as the initial position and the value is $0.50 \mathrm{~m}$. The sprung mass of the quarter whole vehicle was set as $450 \mathrm{~kg}$. The specific parameters of the quarter vehicle are listed in Tab. 4. The angle parameters of the suspension were determined by referring to the angle parameters of kangaroo leg in motion. The detailed parameters are listed in Tab. 5. ${ }^{31}$

An ADAMS simulation model of the quarter vehicle suspension in static balance position was built up, as shown in Fig. 11. 


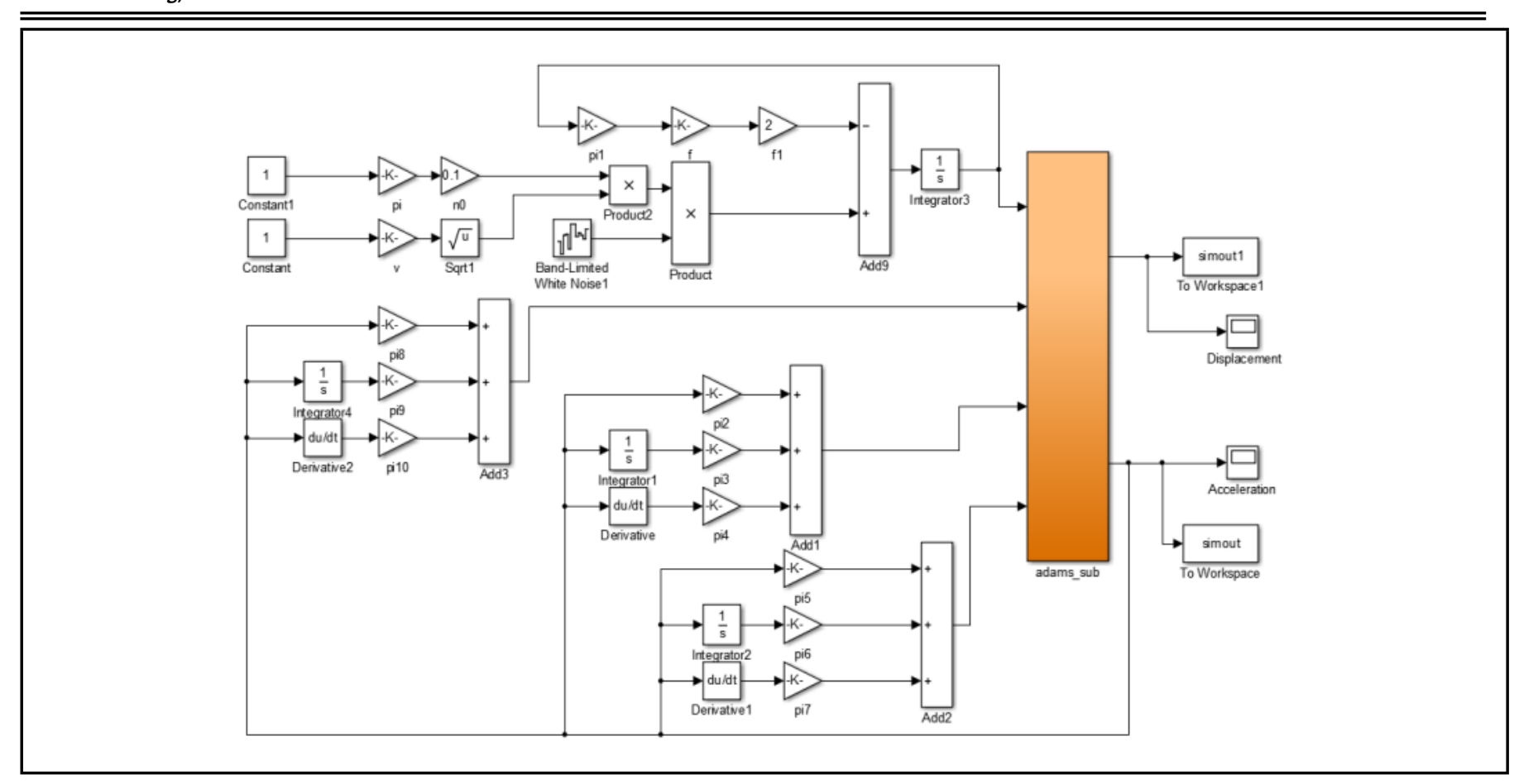

Figure 10. Simulation model of PID control.

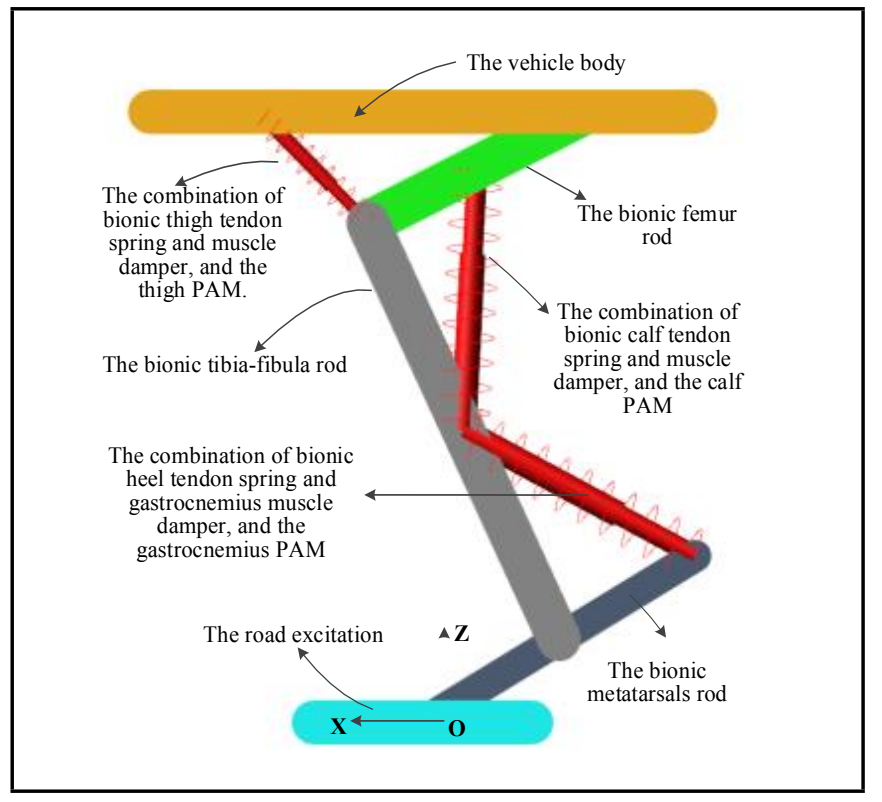

Figure 11. The ADAMS suspension simulation model.

\section{SIMULATION RESULT AND ANALYSIS}

\subsection{Time Domain Response}

A co-simulation model of the quarter vehicle suspension was established based on ADAMS and MATLAB/Simulink, fuzzy control, and PID control were applied to the active control of the suspension.

Three aspects were mainly simulated as follows: (1) the suspension dynamic characteristic; (2) the suspension control characteristic; (3) the suspension velocity characteristic. The time-domain simulations in the passive and active modes were performed under impulse, step, class $\mathrm{C}$ and $\mathrm{D}$ road excitations at different speeds. For the space limitation, only the following simulation results were given as shown in Fig. 12.

The vehicle body dynamic displacement (Fig. 12a), the vehi- cle body acceleration (Fig. 12b), the deformations of the bionic springs and dampers (Fig. 12c) and the angles of the bionic joints (Fig. 12d) in the passive mode were presented under impulse road excitation at $36 \mathrm{~km} / \mathrm{h}$. The vehicle body dynamic displacements in the passive and active modes are shown in Fig. 12e under step road excitation at $36 \mathrm{~km} / \mathrm{h}$ and in Fig. $12 \mathrm{f}$ under class $C$ road excitation at $60 \mathrm{~km} / \mathrm{h}$. The vehicle body dynamic displacements and accelerations in the passive and active modes are given in Figs. 12g, 12i and 12h, 12 j under class D road excitation at $60 \mathrm{~km} / \mathrm{h}$ and $90 \mathrm{~km} / \mathrm{h}$, respectively.

Seen from the suspension dynamic characteristics, it was found that the bionic heel spring deformation (Fig. 12c) was the largest one and the largest angle change occurs in the bionic ankle joint (Fig. 12d) under impulse road excitation, which was similar to the dynamic characteristics of the kangaroo leg, indicating that the bionic design of the suspension structure was feasible and reasonable. Moreover, the upward vehicle body dynamic displacement was reduced by $78.5 \%$ and the downward was reduced by $65.7 \%$, respectively, indicating that the suspension had a good ability of dealing with the impulse excitation in the passive mode (Fig. 12a). The maximum vehicle body acceleration under the upward impulse excitation was half of that under the downward impulse excitation and the minimum vehicle body acceleration under the upward impulse excitation is $87.5 \%$ of that under the downward impulse excitation (Fig. 12b), which indicated that the suspension damping performance under the upward impulse excitation was better than that under the downward impulse excitation.

Furthermore, the simulation parameters of the road excitation and the vehicle speeds are listed in Tab. 6 and the vehicle body dynamic responses are listed in Tab. 7.

Seen from the suspension control characteristics, the root mean squares (RMS) values of the vehicle body dynamic displacement under the fuzzy control and the PID control are basically less than or close to those of the vehicle body dynamic displacement under the passive mode as shown in Figs. 12e, 


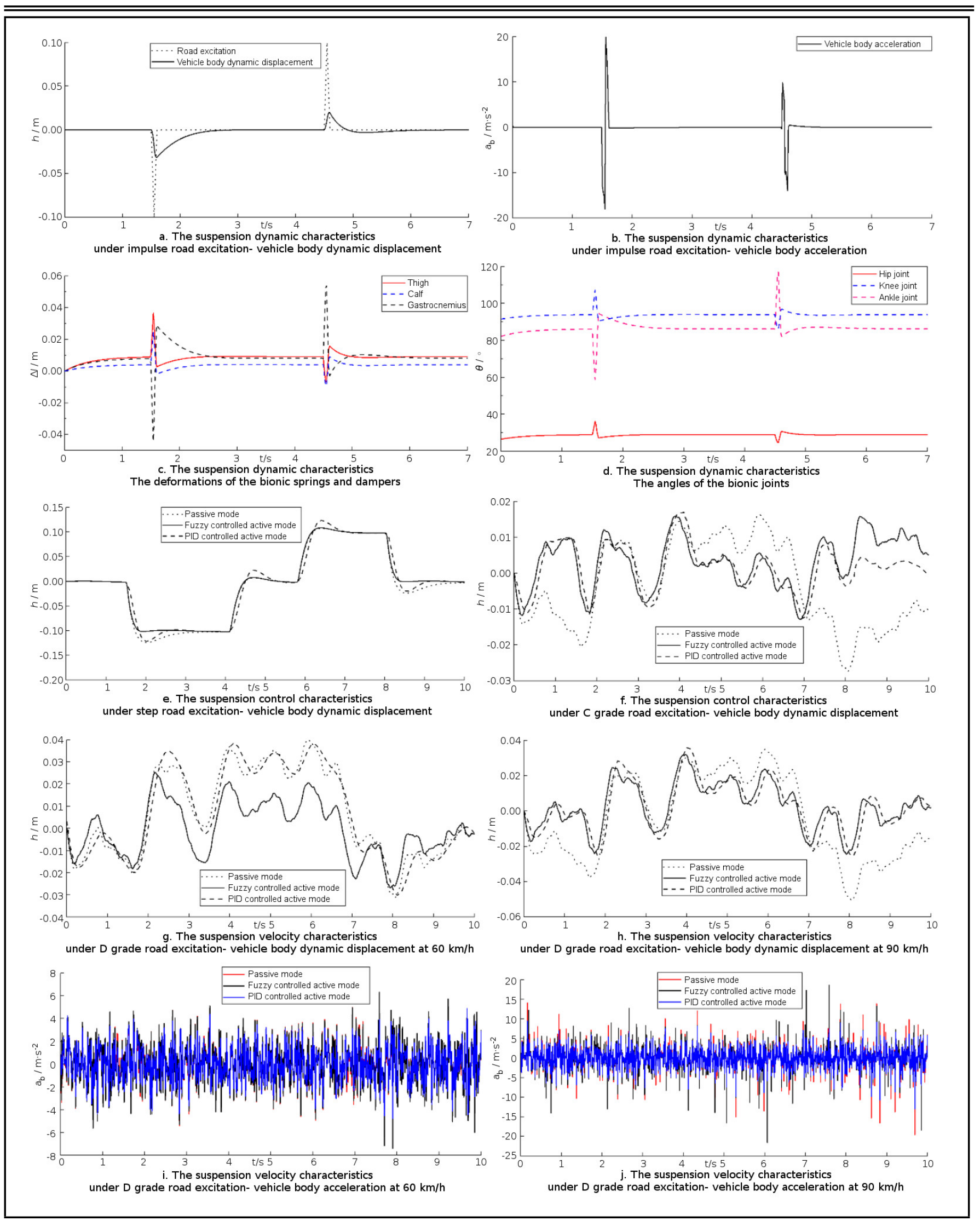

Figure 12. Vertical parameter characteristic simulation of suspension.

$12 \mathrm{f}, 12 \mathrm{~g}$ and $12 \mathrm{~h}$, showing that the fuzzy control and the PID control of the suspension are effective. In addition, the RMS values of the vehicle body acceleration were reduced about 95\% for the three modes (see Figs. $12 \mathrm{i}$ and $12 \mathrm{j}$ and Tab. 7). For the suspension velocity characteristics, the damping per- formance was improved slightly with the increase of the vehicle speed under the same road excitation, and at the same vehicle speed, the damping performance in the three modes was almost similar to each other as shown in Figs. 12g, 12h, $12 \mathrm{i}$ and $12 \mathrm{j}$ and Tab. 7, which demonstrated the good damping 
Table 6. Road excitation and vehicle speeds.

\begin{tabular}{|c|c|c|c|c|c|c|c|}
\hline \multirow[t]{2}{*}{$\begin{array}{l}\text { Road } \\
\text { excitation }\end{array}$} & \multirow[t]{2}{*}{$\begin{array}{l}\text { Speeds/ } \\
\left(\mathrm{km} \cdot \mathrm{h}^{-1}\right)\end{array}$} & \multicolumn{3}{|c|}{ Road excitation/(m) } & \multicolumn{3}{|c|}{$\begin{array}{llll}\begin{array}{l}\text { Accelerations } \\
\text { excitation } /\left(\mathrm{m} \cdot \mathrm{s}^{-2}\right)\end{array} & \text { of } & \text { the } & \text { road } \\
\end{array}$} \\
\hline & & Minimum & Maximum & RMS & Minimum & Maximum & RMS \\
\hline Impulse & 36 & -0.1 & 0.1 & $*$ & -80 & 80 & $*$ \\
\hline Step & 36 & -0.1 & 0.1 & $*$ & -80 & 80 & $*$ \\
\hline Class C & 60 & -0.0189 & 0.0225 & 0.0093 & -63.488 & 68.629 & 18.473 \\
\hline $\mathrm{Cl} / \mathrm{s} \mathrm{D}$ & 60 & -0.0379 & 0.0450 & 0.0186 & -126.976 & 137.259 & 36.964 \\
\hline Crass D & 90 & -0.0464 & 0.0551 & 0.0228 & -155.498 & 168.091 & 45.281 \\
\hline
\end{tabular}

Table 7. Vehicle body dynamic responses.

\begin{tabular}{|c|c|c|c|c|c|c|c|c|c|}
\hline \multirow{2}{*}{$\begin{array}{l}\text { Road } \\
\text { excitation }\end{array}$} & \multirow{2}{*}{ Modes } & \multicolumn{4}{|c|}{ Vehicle body dynamic displacement $h /(\mathrm{m})$} & \multicolumn{4}{|c|}{ Vehicle body acceleration $a_{b} / \mathrm{m} \cdot \mathrm{s}^{-2}$} \\
\hline & & Minimum & Maximum & RMS & $\begin{array}{l}\text { Decrease } \\
\text { percentage } \\
\text { of RMS/ }(\%)\end{array}$ & Minimum & Maximum & RMS & $\begin{array}{l}\text { Decrease } \\
\text { percentage } \\
\text { of RMS/ }(\%)\end{array}$ \\
\hline \multirow{3}{*}{ Impulse } & Passive & -0.0343 & 0.0215 & 0.0026 & $*$ & $*$ & $*$ & $*$ & $*$ \\
\hline & Fuzzy & -0.0333 & 0.0253 & 0.0016 & $*$ & $*$ & $*$ & $*$ & * \\
\hline & PID & -0.0337 & 0.0256 & 0.0020 & $*$ & $*$ & $*$ & $*$ & $*$ \\
\hline \multirow{3}{*}{ Step } & Passive & -0.1239 & 0.1072 & 0.0038 & $*$ & $*$ & $*$ & $*$ & $*$ \\
\hline & Fuzzy & -0.1019 & 0.1084 & 0.0004 & $*$ & $*$ & $*$ & $*$ & $*$ \\
\hline & PID & -0.1250 & 0.1302 & 0.0041 & $*$ & $*$ & $*$ & $*$ & $*$ \\
\hline \multirow{3}{*}{$\begin{array}{l}\text { Class C } \\
(60 \mathrm{~km} / \mathrm{h})\end{array}$} & Passive & -0.0273 & 0.0163 & 0.0030 & 67.74 & -4.546 & 3.549 & 1.046 & 94.23 \\
\hline & Fuzzy & -0.0128 & 0.0159 & 0.0032 & 65.59 & -4.737 & 3.573 & 1.065 & 94.33 \\
\hline & PID & -0.0126 & 0.0189 & 0.0031 & 66.18 & -4.924 & 3.721 & 1.245 & 93.26 \\
\hline \multirow{3}{*}{$\begin{array}{l}\text { Class D } \\
(60 \mathrm{~km} / \mathrm{h})\end{array}$} & Passive & -0.0354 & 0.0372 & 0.0038 & 79.46 & -7.059 & 6.107 & 1.726 & 95.33 \\
\hline & Fuzzy & -0.0276 & 0.0259 & 0.0025 & 86.31 & -7.393 & 6.334 & 1.747 & 95.27 \\
\hline & PID & -0.0334 & 0.0360 & 0.0034 & 81.90 & -5.418 & 4.351 & 1.205 & 96.74 \\
\hline \multirow{3}{*}{$\begin{array}{l}\text { Class D } \\
(90 \mathrm{~km} / \mathrm{h})\end{array}$} & Passive & -0.0433 & 0.0280 & 0.0040 & 82.45 & -19.527 & 14.089 & 2.244 & 95.04 \\
\hline & Fuzzy & -0.0214 & 0.0261 & 0.0024 & 89.47 & -21.563 & 18.582 & 2.284 & 94.95 \\
\hline & PID & -0.0255 & 0.0312 & 0.0029 & 87.34 & -13.768 & 12.160 & 2.006 & 95.57 \\
\hline
\end{tabular}

performance of the suspension.

In addition, relative to the RMS decrease percentage of the road excitation, it can be seen from Tab. 7: (a) under class $\mathrm{C}$ road at the speed of $60 \mathrm{~km} / \mathrm{h}$, the RMS values of the vehicle body dynamic displacement were respectively decreased $67.74 \%, 65.59 \%$, and $66.18 \%$ in the passive, fuzzy control, and PID control modes, indicating the suspension's passive mode had good performance in such a situation; (b) compared with the RMS decrease percentage in the passive mode, the RMS decrease percentages of the fuzzy control and the PID control were increased by $6.85 \%, 7.02 \%, 2.44 \%$, and $4.89 \%$ under class D road at the speed of $60 \mathrm{~km} / \mathrm{h}$ and $90 \mathrm{~km} / \mathrm{h}$, respectively, showing that the effect of fuzzy control was slightly better than PID control in the active mode; (c) with the increases of road grade and vehicle speed, the RMS decreased percentage as the suspension response increased, explaining that the suspension had good adaptability. As a whole, the performances of the PAM bionic kangaroo leg suspension under the fuzzy control mode and the PID control mode were both better than that of the suspension under the passive mode.

\subsection{Frequency Responses}

The PAM bionic kangaroo leg suspension is a non-linear suspension system, its active mode was realized through cosimulation with MATLAB. The transfer function of suspension system cannot be directly solved, and its frequency response was difficult to obtain. Therefore, the approximate estimation method was adopted to analyse the frequency response of PAM bionic kangaroo leg suspension in the passive and active modes. ${ }^{32,33}$ The method is as follows:

(1) Taking the sinusoidal excitation of $K$ periods as the road input, its equation is:

$$
x_{r}(t)=A \sin (2 \pi f t)
$$

where $A \in[1,5] \mathrm{cm} ; f \in\left[f_{-}, f^{-}\right] \subseteq[0.1,25] \mathrm{Hz} ; t \in[0, T]$; the signal duration $T=K / f, K$ was the periodic numbers of the sinusoidal signal, generally $K=10$.

(2) Measure the output signal $z(t)$ of the suspension system.

(3) Calculate the transmissibility of vibration damping performance index $H_{a}(f)$ :

$$
H_{a}(f)=\sqrt{\frac{\frac{1}{T} \int_{0}^{T} z^{2}(t) \mathrm{dt}}{\frac{1}{T} \int_{0}^{T} x_{r}^{2}(t) \mathrm{dt}}}
$$

In order to eliminate the calculation error caused by the system in an unstable state, the initial part of the output signal was usually truncated.

(4) Change the frequency of the sinusoidal excitation and repeat steps (1)-(3) to get a frequency response in the desired frequency range.

In this paper, the frequency response of the suspension was measured according to the above method, and $A=1 \mathrm{~cm}, K=10$, using MATLAB to perform calculations every $0.1 \mathrm{~Hz}$ in the range of $0.1 \mathrm{~Hz}-10 \mathrm{~Hz}$, and every $1 \mathrm{~Hz}$ from $10 \mathrm{~Hz}$ to $25 \mathrm{~Hz}$. The response of the vehicle body acceleration transmissibility and the suspension dynamic deflection transmissibility in the frequency domain were measured. Figure 13 shows the vibration damping performance index approximate estimation results of the bionic kangaroo leg suspension.

Figure 13a is the frequency response of the vehicle body acceleration transmissibility, and Fig. 13b is the frequency response of the suspension dynamic deflection transmissibility. In the resonance region of the vehicle body, the fuzzy control and the PID control effectively reduced the transmissibility of various performance indexes. In low frequency range $(0-1 \mathrm{~Hz})$, the performance indexes under the fuzzy control and the PID control were equivalent to the passive mode of the suspension, but the PID control was slightly better than the fuzzy control. 


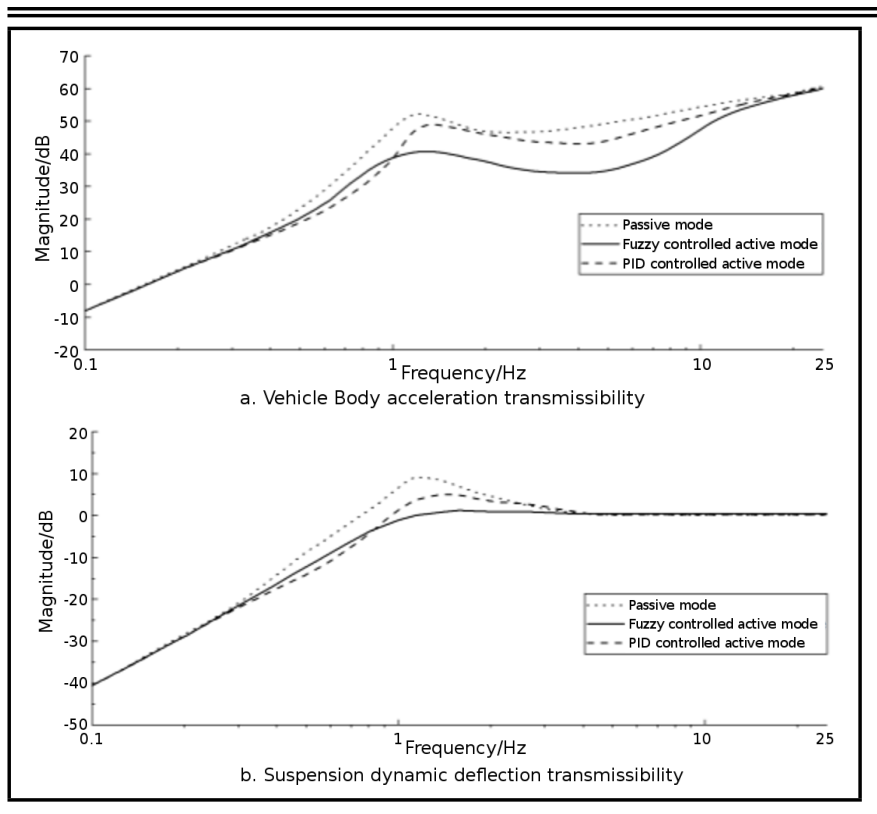

Figure 13. Frequency response of suspension.

In medium frequency range $(1-10 \mathrm{~Hz})$, the fuzzy control and the PID control both significantly reduced the transmissibility of body acceleration, and the fuzzy control was better than the PID control, the suspension dynamic deflection was not greatly affected by the control strategy. In high frequency range (10 $25 \mathrm{~Hz}$ ), the fuzzy control and the PID control were equivalent to the passive mode of the suspension, the dynamic deflection of the suspension was less affected by the control strategy.

The frequency response results of the suspension showed that the fuzzy control and the PID control can reduce the transmissibility of various performance indexes of the suspension; the control effects of the two control methods had their advantages and disadvantages in different frequency ranges, and they were better than those of the passive mode in most frequency bands. In addition, the suspension dynamic deflection was not greatly affected by the control strategy.

\section{CONCLUSIONS}

A PAM kangaroo leg bionic suspension is proposed by analysing and refining the kangaroo leg structure and functions. The suspension theoretical modelling is performed by the Lagrange's Equations. The co-simulation of ADAMS and MATLAB is accomplished under the fuzzy control and the PID control. The dynamic response characteristics of the suspension in the passive and active modes are figured out and analysed in the time and frequency domains. The following conclusions are obtained.

(1) Seen from the dynamic characteristic, the velocity characteristic and the control characteristic of the suspension, the proposed PAM bionic kangaroo leg suspension can well cope with the vibration impact under different road excitations and different speeds, suggesting that the suspension structure design is reasonable, the damping performance is satisfactory and the fuzzy control and the PID control are effective.

(2) The suspension performance in the active mode is better than that of the passive mode. Furthermore, some amplitudes in the passive mode are amplified under the concave road excitation, indicating that there is further optimization space for this PAM bionic kangaroo leg suspension.
(3) For the frequency response, the fuzzy control and the PID control are more effective to reduce the transmissibility of the suspension in the medium frequency ranges $(1-10 \mathrm{~Hz})$, and their control effects are also better than those of the passive mode in most frequency bands $(0-25 \mathrm{~Hz})$. Moreover, the suspension dynamic deflection is not obviously affected by different control strategy.

\section{ACKNOWLEDGEMENTS}

This research was financially supported by National Natural Science Foundation of China (Grant No.51305288,51805347), Natural Science Foundation of Shanxi Province (Grant No. 201901D111245,201901D211293), the Fund for Shanxi "1331 Project" Key Subjects Construction (Grant No.1331KSC) and Shanxi Science and Technology Foundation Platform Construction Projects (Grant No.201805D121005).

\section{REFERENCES}

1 Ishida, Y. Recent development of the passive vibration control method, Mechanical Systems and Signal Processing, 29, 2-18, (2012). https://dx.doi.org/10.1016/j.ymssp.2011.12.005

2 Rizvi, S. M. H., Abid, M., Khan, A. Q., Satti, S. G., and Latif, J. $\mathrm{H} \infty$ control of 8 degrees of freedom vehicle active suspension system, Journal of King Saud University Science, 30 (2), 161-169, (2018). https://dx.doi.org/10.1016/j.jksues.2016.02.004

3 Hrovat, D. Survey of advanced suspension developments and related optimal control applications, Automatica, 33 (10), 1781-1817, (1997). https://dx.doi.org/10.1016/s00051098(97)00101-5

4 Papaioannou, G., and Koulocheris, D. An approach for minimizing the number of objective functions in the optimization of vehicle suspension systems, Journal of Sound and Vibration, 435, 149-169, (2018). https://dx.doi.org/10.1016/j.jsv.2018.08.009

5 He, J., and Gao, F. Type synthesis for bionic quadruped walking robots, Journal of Bionic Engineering, 12 (4), 527-538, (2015). https://dx.doi.org/10.1016/S16726529(14)60143-8

6 Shen, X. R. Nonlinear model-based control of pneumatic artificial muscle servo systems, Control Engineering Practice, 18 (3), 311-317, (2010). https://dx.doi.org/10.1016/j.conengprac.2009.11.010

7 Biewener, A. A. Locomotor design for acceleration versus elastic energy storage in kangaroo rat hopping, Journal of Biomechanics, 20 (9), 896, (1987). https://dx.doi.org/10.1016/0021-9290(87)90174-6

8 Dawson, T. Energetic cost of locomotion in kangaroos, Nature, 246, 134-313, (1973). https://dx.doi.org/10.1038/246313a0

9 Hanan, U. B., Weiss, A., and Zaitsev, V. Jumping efficiency of small creatures and its applicability in robotics, Procedia Manufacturing, 21, 243-250, (2018). https://dx.doi.org/10.1016/j.promfg.2018.02.117 
${ }^{10}$ Chen, D. S., Yin, J. M., Zhao, K., Zheng, W. J., and Wang, T. M. Bionic mechanism and kinematics analysis of hopping robot inspired by locust jumping, Journal of Bionic Engineering, 8 (4), 429-439, (2011). https://dx.doi.org/10.1016/S1672-6529(11)60048-6

11 O'Connor, S. M., Dawson, T. J., Kram, R., and Donelan, J. M. The kangaroo's tail propels and powers pentapedal locomotion, Biology Letters, 10 (7), (2014). https://dx.doi.org/10.1098/rsbl.2014.0381

12 Kar, D. C., Kurien Issac, K., and Jayarajan, K. Gaits and energetics in terrestrial legged locomotion, Mechanism and Machine Theory, 38 (4), 355-366, (2003). https://dx.doi.org/10.1016/S0094-114X(02)00124-6

${ }^{13}$ Festo, A. G \& Co. KG, BionicKangaroo energy-efficient jump kinematics based on a natural model, Retrieved from http://www.festo.com/net/SupportPortal/Files/334102/ Festo_BionicKangaroo_de.pdf, (Accessed January 8, 2019).

${ }^{14}$ Knut, G., Sebastian, H., Alexander, H., Nadine, K., Nina, G., and Elias, K. Control design for a bionic kangaroo, Control Engineering Practice, 42, 106-117, (2015). https://dx.doi.org/10.1016/j.conengprac.2015.05.005

15 K, K. G., and Pathak, P. M. Dynamic modelling \& simulation of a four-legged jumping robot with compliant legs, Robotics and Autonomous Systems, 61 (3), 221-228, (2013). https://dx.doi.org/10.1016/j.robot.2012.09.025

${ }^{16}$ Ge, W. J., Shen, Y. W., and Yang, F. Kinematics of kangaroo robot jumping gait, Chinese Journal of Mechanical Engineering, 5, 22-26, (2006). https://dx.doi.org/cnki:sun:jxxb.0.2006-05-005

17 Zuo, G. Y., and Liu, X. Stable jumping control of bionic kangaroo robot using spring-loaded inverted pendulum model, Control Theory \& Applications, 35 (8), 1151-1158, (2018). https://dx.doi.org/10.7641/CTA.2018.70379

18 Liu, G. H., Lin, H. Y., Lin, H. Y., Chen, S. T., and Lin, P. C. A Bio-Inspired Hopping Kangaroo Robot with an Active Tail, Journal of Bionic Engineering, 11 (4), 541-555, (2014). https://dx.doi.org/10.1016/S1672-6529(14)600664

19 Iida, F., Rummel, J., and Seyfarth, A. Bipedal walking and running with spring-like biarticular muscles, Journal of Biomechanics, 41 (3), 656-667, (2008). https://dx.doi.org/10.1016/j.jbiomech.2007.09.033

20 Souchet, R. Continuum mechanics and Lagrange equations with generalised coordinates, International Journal of Engineering Science, 76, 27-33, (2014). https://dx.doi.org/10.1016/j.ijengsci.2013.11.017

21 Kalita, B., and Dwivedy, S. K. Nonlinear dynamics of a parametrically excited pneumatic artificial muscle (PAM) actuator with simultaneous resonance condition, Mechanism and Machine Theory, 135, 281-297, (2019). https://dx.doi.org/10.1016/j.mechmachtheory.2019.01.031

22 Al-Fahaam, H., Davis, S., and Nefti-Meziani, S. The design and mathematical modelling of novel extensor bending pneumatic artificial muscles (EBPAMs) for soft exoskeletons, Robotics and Autonomous Systems, 99, 63-74, (2018). https://dx.doi.org/10.1016/j.robot.2017.10.010

23 Wickramatunge, K. C., and Leephakpreeda, T. Study on mechanical behaviours of pneumatic artificial muscle, International Journal of Engineering Science, 48 (2), 188-198, (2010). https://dx.doi.org/10.1016/j.ijengsci.2009.08.001

24 Jahanabadi, H., Mailah, M., Md Zain, M. Z., and Hooi, H. M. Active Force with Fuzzy Logic Control of a Two-Link Arm Driven by Pneumatic Artificial Muscles, Journal of Bionic Engineering, 8 (4), 474-484, (2011). https://dx.doi.org/10.1016/S1672-6529(11)60053-X

${ }^{25}$ Festo AG \& Co. KG, DMSP parameters, Retrieved from https://www.festo.com.cn/cat/zh-cn_cn/products_DMSP, (Accessed April 15, 2019).

${ }^{26}$ Sezer, S., Cetin, S., and Atalay, A. E. Application of self-tuning fuzzy logic control to full railway vehicle model, Procedia Computer Science, 6, 487-492, (2011). https://dx.doi.org/10.1016/j.procs.2011.08.090

27 Adnan, M. M., Sarkheyli, A., Zain, A. M., and Haron, H. Fuzzy logic for modelling machining process: a review, Artificial Intelligence, 43 (3), 345-379, (2015). https://dx.doi.org/10.1007/s10462-012-9381-8

${ }^{28}$ Lee, C. C. Fuzzy logic in control systems: fuzzy logic controller, IEEE Transactions on Systems Man Cybernetics-Systems, 20 (2), 404-418, (1990). https://dx.doi.org/10.1109/21.52551

29 Gandhi, P., Adarsh, S., and Ramachandran, K. I. Performance Analysis of Half Car Suspension Model with 4 DOF using PID, LQR, FUZZY and ANFIS Controllers, Procedia Computer Science, 115, 2-13, (2017). https://dx.doi.org/10.1016/j.procs.2017.09.070

30 Kasemi, B., Asan, G. A., Muthalif, Mahbubur Rashid, M., and Fathima, S. Fuzzy-PID Controller for SemiActive Vibration Control Using Magnetorheological Fluid Damper, Procedia Engineering, 41, 1221-1227, (2012). https://dx.doi.org/10.1016/j.proeng.2012.07.304

31 Kurebwa, J., and Mushiri, T. Design and Simulation of an Integrated Steering System for Allpurpose Sport Utility Vehicles (SUVs) - Case for Toyota, Procedia Manufacturing, 35, 56-74, (2019). https://dx.doi.org/10.1016/j.promfg.2019.07.002

32 Zhang, L., Zhang, J. Q., Luo, T., Bi, Z. D., and Yao, J. A Research on Comprehensive Performance Evaluation Method for Vehicle Suspension System, Automotive Engineering, 38 (12), 1494-1499, (2016). https://dx.doi.org/10.3969/j.issn.1000-680X.2016.12.015

33 Wang, G., Chen, C. Z., and Yu, S. B. Robust nonfragile finite-frequency $\mathrm{H} \infty$ static output-feedback control for active suspension systems, Mechanical Systems and Signal Processing, 91, 41-56, (2017). https://dx.doi.org/10.1016/j.ymssp.2016.12.039 